\title{
Inequalities related to the third Jackson $q$-Bessel function of order zero
}

\author{
Hédi Elmonser ${ }^{1 *}$, Mouna Sellami² and Ahmed Fitouhi ${ }^{3}$
}

\section{"Correspondence:}

monseur2004@yahoo.fr

${ }^{1}$ College of Science and Human

Studies at Hotat Sudair, Majmaah

University, Riyadh, Saudi Arabia

Full list of author information is

available at the end of the article

\begin{abstract}
In Bettaibi and Bouzeffour (J. Math. Anal. Appl. 342:1203-1219, 2008), some properties of the third Jackson $q$-Bessel function of order zero were established. This paper is devoted to studying the $q$-convolution product by using a $q$-integral representation of the related $q$-translation.

The central part of this work is first to study the related $q$-heat semi-group and its hypercontractivity and second to specify the $q$-analogue of the Wiener algebra.
\end{abstract}

\section{Introduction: notations and preliminaries}

\subsection{Introduction}

In harmonic analysis the positivity of the translation operator is crucial. It plays a central role in establishing some useful results such as the properties of the convolution product.

In contrast to the classical theory, the positivity of the translation operator associated to the normalized $q$-Bessel function of order $\alpha$ is not clear at this stage. In fact it is still an open conjecture to find $q \in[0,1]$ and $\alpha$ which assure the positivity of the related translation. For $\alpha=-1 / 2$, it was proved that the $q$-translation is not positive for all $q \in[0,1]$ (see [1]). However, for $\alpha=0$, the authors proved (see [2]) that the $q$-translation is positive for all $q \in[0,1]$. This fact helps us to study the harmonic analysis associated to the third Jackson $q$-Bessel function of order zero and to establish some important inequalities.

This paper is organized as follows. In Section 2 we begin by summarizing some statements concerning the $q$-translation operator $T_{x, q}$ studied in [2]. Then we prove some facts about the positivity and the $x$-continuity of $T_{x, q}$ for an appropriate space and we give an integral representation.

In Section 3, we recall some basic properties of the $q$-convolution product cited in [2]. Then we establish some results of density.

In Section 4, we study the $q$-Fourier Bessel transform $\mathcal{F}_{q}(f)$ : after recalling some results in [2] and by the use of the inversion formula, we prove that we can extend the definition of $\mathcal{F}_{q}(f)$ to $L_{q}^{1}\left(\mathbb{R}_{q,+}, x d_{q} x\right)$ and by density to $L_{q}^{p}\left(\mathbb{R}_{q,+}, x d_{q} x\right), 1<p \leq 2$.

Sections 5 and 6 are reserved to study the $q$-analogue of some well-known results associated to the heat semi-group and the Wiener algebra.

\subsection{Notations and preliminaries}

We recall some usual notions and notations used in the $q$-theory (see [3]). We refer to the book by Gasper and Rahmen [3] for the definitions, notations and properties of the $q$ shifted factorials and the $q$-hypergeometric functions. Throughout this paper, we assume 
that $q \in] 0,1[$ and we note

$$
[x]_{q}=\frac{1-q^{x}}{1-q}, \quad x \in \mathbb{C} .
$$

The $q$-derivatives $D_{q} f$ and $D_{q}^{+} f$ of a function $f$ are given by

$$
\left(D_{q} f\right)(x)=\frac{f(x)-f(q x)}{(1-q) x}, \quad\left(D_{q}^{+} f\right)(x)=\frac{f\left(q^{-1} x\right)-f(x)}{(1-q) x} \quad \text { if } x \neq 0 \text {, }
$$

$\left(D_{q} f\right)(0)=f^{\prime}(0)$ and $\left(D_{q}^{+} f\right)(0)=q^{-1} f^{\prime}(0)$ provided $f^{\prime}(0)$ exists.

Using these two derivatives, we put

$$
\Delta_{q}=\frac{(1-q)^{2}}{x} D_{q}\left[x D_{q}^{+}\right]
$$

The $q$-Jackson integrals from 0 to $a$ and from 0 to $\infty$ are defined by (see [4])

$$
\begin{aligned}
& \int_{0}^{a} f(x) d_{q} x=(1-q) a \sum_{n=0}^{\infty} f\left(a q^{n}\right) q^{n} \\
& \int_{0}^{\infty} f(x) d_{q} x=(1-q) \sum_{n=-\infty}^{\infty} f\left(q^{n}\right) q^{n}
\end{aligned}
$$

provided the sums converge absolutely.

The $q$-Jackson integral in a generic interval $[a, b]$ is given by (see [4])

$$
\int_{a}^{b} f(x) d_{q} x=\int_{0}^{b} f(x) d_{q} x-\int_{0}^{a} f(x) d_{q} x
$$

We recall that the $q$-hypergeometric function ${ }_{1} \varphi_{1}$ satisfies the following properties (see [5] or [6]):

(1) For all $w, z \in \mathbb{C}$, we have

$$
(w, q)_{\infty 1} \varphi_{1}(0 ; w ; q ; z)=(z, q)_{\infty 1} \varphi_{1}(0 ; z ; q ; w) .
$$

(2) For $n \in \mathbb{N}$ and $z \in \mathbb{C}$, we have

$$
\left(q^{1-n} ; q\right)_{\infty^{1}} \varphi_{1}\left(0 ; q^{1-n} ; q ; z\right)=(-1)^{n} q^{\frac{n(n-1)}{2}} z^{n}\left(q^{n+1} ; q\right)_{\infty^{1}} \varphi_{1}\left(0 ; q^{n+1} ; q ; q^{n} z\right)
$$

(3) Both sides of (6) are majorized by

$$
(-z ; q)_{\infty}(-w ; q)_{\infty} \quad \text { and } \quad q^{\frac{n(n-1)}{2}}|z|^{n}(-|z| ; q)_{\infty}(-q ; q)_{\infty} \quad \text { if } w=q^{1-n}(n \in \mathbb{N})
$$

In [6] Koornwinder and Swarttouw, in order to study a $q$-analogue of the Hankel transform and to give its inversion formula and a Plancherel formula, defined the third Jackson $q$-Bessel function using the $q$-hypergeometric function ${ }_{1} \varphi_{1}$ as follows:

$$
J_{\alpha}\left(z ; q^{2}\right)=\frac{z^{\alpha}\left(q^{2 \alpha+2} ; q^{2}\right)_{\infty}}{\left(q^{2} ; q^{2}\right)_{\infty}} \varphi_{1}\left(0 ; q^{2 \alpha+2} ; q^{2}, q^{2} z^{2}\right) .
$$


They proved the following orthogonality relation:

$$
\sum_{k=-\infty}^{\infty} q^{2 k} q^{n+m} J_{n+k}\left(x ; q^{2}\right) J_{m+k}\left(x ; q^{2}\right)=\delta_{n, m}, \quad|x|<q^{-1}, n, m \in \mathbb{Z}
$$

In [7], and more generally in [8], the authors gave the following $q$-analogue of Graf's addition formula by the use of an analytic approach:

$$
J_{\nu}\left(R q^{(y+z+v)} ; q^{2}\right) J_{x-v}\left(q^{z} ; q^{2}\right)=\sum_{k=-\infty}^{\infty} J_{v}\left(R q^{(y+x+k)} ; q^{2}\right) J_{v+k}\left(R q^{y+v+k} ; q^{2}\right) J_{x}\left(q^{(z-k)} ; q^{2}\right)
$$

where $z \in \mathbb{Z}, x, y, v \in \mathbb{C}$ satisfy $q^{2(1+\mathcal{R}(x)+\mathcal{R}(y))}|R|^{2}<1, \mathcal{R}(x)>-1$ and $R \neq 0$. We have the following behavior (see [2]).

Lemma 1 For $\alpha \geq-\frac{1}{2}$ and $x \in \mathbb{R}_{q,+}=\left\{q^{n}: n \in \mathbb{Z}\right\}$, we have

(1)

$$
\left|J_{\alpha}\left(x ; q^{2}\right)\right| \leq \frac{\left(-q^{2} ; q^{2}\right)_{\infty}\left(-q^{2^{(\alpha+1)}} ; q^{2}\right)_{\infty}}{\left(q^{2} ; q^{2}\right)_{\infty}} \begin{cases}x^{\alpha} & \text { if } x \leq \frac{q}{1-q} \\ q^{\left(\frac{\log (x)}{\log q}\right)^{2}} & \text { if } x \geq \frac{q}{1-q}\end{cases}
$$

(2) For all $v \in \mathbb{R}$, we have $J_{\alpha}\left(x ; q^{2}\right)=o\left(x^{-v}\right)$ as $x \rightarrow+\infty$.

In particular, we have $\lim _{x \rightarrow+\infty} J_{\alpha}\left(x ; q^{2}\right)=0$.

(3) $D_{q}^{+}\left(x^{-\alpha} J_{\alpha}\left(x ; q^{2}\right)\right)=-(1-q)^{-1} x^{-\alpha} J_{\alpha+1}\left(x ; q^{2}\right)$.

In literature, some authors (see [5]) developed some elements of $q$-harmonic analysis related to the normalized $q-j_{\alpha}$ function using a transmutation operator.

In this paper, we are concerned with $J_{0}\left(x ; q^{2}\right)$, the third Jackson $q$-Bessel function of order zero. We construct a product formula for this function leading to a positive $q$-translation which is necessary and constructive for some applications.

It is well known (see [5], Prop. 1) that for all $\lambda \in \mathbb{C}$, the function

$$
J_{0}\left(\lambda x ; q^{2}\right)=\sum_{k=0}^{\infty} \frac{(-1)^{k} q^{k(k+1)} \lambda^{2 k} x^{2 k}}{\left(q^{2} ; q^{2}\right)_{k}^{2}}
$$

is the solution of the $q$-problem

$$
\left\{\begin{array}{l}
\Delta_{q} y(x)+\lambda^{2} y(x)=0 \\
y(0)=1, \quad y^{\prime}(0)=0
\end{array}\right.
$$

We need the following spaces and sets:

- $\mathbb{R}_{q}=\left\{ \pm q^{n}: n \in \mathbb{Z}\right\}, \mathbb{R}_{q,+}=\left\{q^{n}: n \in \mathbb{Z}\right\}$ and $\widetilde{\mathbb{R}}_{q,+}=\mathbb{R}_{q,+} \cup\{0\}$.

- $\mathcal{S}_{* q}\left(\mathbb{R}_{q,+}\right)$ the space of restrictions on $\mathbb{R}_{q,+}$ of even functions $f$ such that for all $m, n \in \mathbb{N}$, we have $\sup _{x \in \mathbb{R}_{q,+}}\left|x^{2 m} \Delta_{q}^{n}(f)(x)\right|<\infty$ and for all $n \in \mathbb{N}$, we have $\left(D_{q}^{+}\left(\Delta_{q}^{n}(f)\right)\right)(x) \rightarrow 0$ as $x \downarrow 0$ in $\mathbb{R}_{q,+}$.

- $\mathcal{D}_{* q}\left(\mathbb{R}_{q,+}\right)$ the space of restrictions on $\mathbb{R}_{q,+}$ of even functions with bounded support such that for all $n \in \mathbb{N}$, we have $\left(D_{q}^{+}\left(\Delta_{q}^{n} f\right)\right) \rightarrow 0$ as $x \downarrow 0$ in $\mathbb{R}_{q,+}$. 
- $\mathcal{C}_{* q, 0}\left(\mathbb{R}_{q,+}\right)$ the space of restrictions on $\mathbb{R}_{q,+}$ of even functions, for which $f(x) \rightarrow 0$ as $x \rightarrow+\infty$ in $\mathbb{R}_{q,+}$ and $f(x) \rightarrow 0$ as $x \downarrow 0$ in $\mathbb{R}_{q,+}$, equipped with the norm

$$
\|f\|_{\infty, q}=\sup _{x \in \mathbb{R}_{q,+}}|f(x)|
$$

- $\mathcal{C}_{* q, b}\left(\widetilde{\mathbb{R}}_{q,+}\right)$ the space of restrictions on $\widetilde{\mathbb{R}}_{q,+}$ of even functions for which $f(x) \rightarrow f(0)$ as $x \downarrow 0$ in $\mathbb{R}_{q,+}$ and

$$
\|f\|_{\infty, q}=\sup _{x \in \widetilde{\mathbb{R}}_{q,+}}|f(x)|<\infty
$$

- $L_{q}^{p}\left(\mathbb{R}_{q,+}, x d_{q} x\right), p>0$, the set of all functions defined on $\mathbb{R}_{q,+}$ such that

$$
\|f\|_{p, q}=\left\{\int_{0}^{\infty}|f(x)|^{p} x d_{q} x\right\}^{\frac{1}{p}}<\infty
$$

- $L_{q}^{\infty}\left(\mathbb{R}_{q,+}\right)$, the set of all functions defined on $\mathbb{R}_{q,+}$ such that

$$
\|f\|_{\infty, q}=\sup _{x \in \mathbb{R}_{q,+}}|f(x)|<\infty .
$$

\section{A q-generalized translation}

In [2], using the kernel

$$
K\left(q^{m}, q^{n}, q^{k}\right)=\left[J_{m-k}\left(q^{n-k} ; q^{2}\right)\right]^{2}, \quad m, n, k \in \mathbb{Z},
$$

the authors defined the $q$-generalized translation as

$$
\left\{\begin{array}{l}
T_{0, q} f=f \\
T_{x, q} f(y)=\sum_{k=-\infty}^{\infty} K\left(x, y, q^{k}\right) f\left(q^{k}\right), \quad x, y \in \mathbb{R}_{q,+},
\end{array}\right.
$$

provided the sum converges.

The kernel $K$ satisfies the following properties.

For $m, n, k \in \mathbb{Z}$, we have

(1)

$$
0 \leq K\left(q^{m}, q^{n}, q^{k}\right) \leq \frac{\left(-q^{2(1+n-k)},-q^{2} ; q^{2}\right)_{\infty}^{2}}{\left(q^{2} ; q^{2}\right)_{\infty}^{2}} \begin{cases}q^{2(m-k)(n-k)} & \text { if } m \geq k \\ q^{2(m-k)(m-n-1)} & \text { if } m \leq k\end{cases}
$$

(2)

$$
K\left(q^{m}, q^{n}, q^{k}\right)=K\left(q^{n}, q^{m}, q^{k}\right)
$$

(3)

$$
K\left(q^{m}, q^{n}, q^{k}\right)=q^{2(k-n)} K\left(q^{m}, q^{k}, q^{n}\right) .
$$


(4)

$$
q^{2 m+2 n} K\left(q^{m}, q^{n}, q^{k}\right) \text { is symmetric in } n, m \text { and } k \text {. }
$$

(5)

$$
\sum_{n=-\infty}^{\infty} q^{2(n-k)} K\left(q^{n}, q^{m}, q^{k}\right)=1
$$

(6)

$$
\forall m, n, k \in \mathbb{Z}, \quad 0 \leq K\left(q^{m}, q^{n}, q^{k}\right) \leq \min \left\{1, q^{2|k-n|}, q^{2|k-m|}, q^{2|n-m|}\right\} .
$$

(7)

$$
K\left(q^{m+r}, q^{n+r}, q^{k+r}\right)=K\left(q^{m}, q^{n}, q^{k}\right), \quad r \in \mathbb{Z} .
$$

It was shown in [2] that the generalized $q$-translation satisfies the following results.

\section{Proposition 1}

(1) The q-generalized translation is positive.

(2) $T_{x, q} f(y)=T_{y, q} f(x), x, y \in \widetilde{\mathbb{R}}_{q,+}$.

(3) For $f \in L^{1}\left(\mathbb{R}_{q,+}, x d_{q} x\right), y \in \mathbb{R}_{q,+}, T_{0, q} f(y)=\lim _{n \rightarrow+\infty} T_{q^{n}, q} f(y), y \in \mathbb{R}_{q,+}$.

(4) $T_{x, q} J_{0}\left(\cdot ; q^{2}\right)(y)=J_{0}\left(x ; q^{2}\right) J_{0}\left(y ; q^{2}\right), x, y \in \mathbb{R}_{q,+}$.

Proposition 2 For $f, g \in L^{1}\left(\mathbb{R}_{q,+}, x d_{q} x\right)$, we have for all $x \in \mathbb{R}_{q,+}$,

(1) $\int_{0}^{\infty} T_{x, q} f(y) y d_{q} y=\int_{0}^{\infty} f(y) y d_{q} y$.

(2) $\int_{0}^{\infty} T_{x, q} f(y) g(y) y d_{q} y=\int_{0}^{\infty} f(y) T_{x, q} g(y) y d_{q} y$.

Now, we put, for $x, y, t \in \mathbb{R}_{q,+}$

$$
W(x, y, t)=\frac{K(x, y, t)}{(1-q) t^{2}} .
$$

Using the proprieties of the kernel $K$ and the definition of the generalized $q$-translation, one can state the following results.

\section{Proposition 3}

(1)

$$
W(x, y, t) \geq 0 .
$$

(2)

$$
W(x, y, t)=W(y, x, t)=W(x, t, y), \quad \forall x, y, t \in \mathbb{R}_{q,+} .
$$


(3)

$$
\int_{0}^{+\infty} W(x, y, t) t d_{q} t=1
$$

(4) For $x, y \in \mathbb{R}_{q,+}$, we have

$$
T_{x, q} f(y)=\int_{0}^{+\infty} f(t) W(x, y, t) t d_{q} t
$$

The following result is useful for the remainder.

\section{Proposition 4}

(1) For $f \in L_{q}^{p}\left(\mathbb{R}_{q,+}, x d_{q} x\right), p \geq 1$ and $x \in \widetilde{\mathbb{R}}_{q,+}$, we have $T_{x, q}(f) \in L^{p}\left(\mathbb{R}_{q,+}, x d_{q} x\right)$ and

$$
\left\|T_{x, q}(f)\right\|_{p, q} \leq\|f\|_{p, q} .
$$

(2) For $f \in L_{q}^{\infty}\left(\mathbb{R}_{q,+}\right)$ and $x \in \widetilde{\mathbb{R}}_{q,+}$, we have $T_{x, q}(f) \in L_{q}^{\infty}\left(\mathbb{R}_{q,+}\right)$ and

$$
\left\|T_{x, q}(f)\right\|_{\infty, q} \leq\|f\|_{\infty, q} .
$$

Proof The case $x=0$ is evident.

- If $p \in[1,+\infty[$.

Using (28) and the $q$-Hölder inequality, we deduce, for $x, y \in \mathbb{R}_{q,+}$,

$$
\left(\int_{0}^{+\infty}|f(t)| W(x, y, t) t d_{q} t\right)^{p} \leq \int_{0}^{+\infty}|f(t)|^{p} W(x, y, t) t d_{q} t
$$

Applying Fubini-Tonelli's theorem and the relations (27) and (28), we obtain

$$
\left\|T_{x, q}(f)\right\|_{p, q}^{p} \leq \int_{0}^{+\infty}|f(t)|^{p}\left(\int_{0}^{+\infty} W(x, y, t) y d_{q} y\right) t d_{q} t=\|f\|_{p, q}^{p} .
$$

- If $p=+\infty$.

$\forall x \in \mathbb{R}_{q,+}, \forall y \in \mathbb{R}_{q,+}$,

$$
\left|T_{x, q}(f)(y)\right| \leq \int_{0}^{+\infty}|f(t)| W(x, y, t) t d_{q} t \leq\|f\|_{\infty, q} \int_{0}^{+\infty} W(x, y, t) t d_{q} t=\|f\|_{\infty, q}
$$

which achieves the proof.

Corollary 1 For $f \in L_{q}^{p}\left(\mathbb{R}_{q,+}, x d_{q} x\right), p \geq 1$, the mapping $x \rightarrow T_{x, q}(f)$ from $\widetilde{\mathbb{R}}_{q,+}$ into $L_{q}^{p}\left(\mathbb{R}_{q,+}, x d_{q} x\right)$ is continuous at 0 , i.e.,

$$
\lim _{x \rightarrow 0}\left\|T_{x, q}(f)-f\right\|_{p, q}=0 .
$$

For $f \in L_{q}^{\infty}\left(\mathbb{R}_{q,+}\right)$, the mapping $x \rightarrow T_{x, q}(f)$ from $\widetilde{\mathbb{R}}_{q,+}$ into $L_{q}^{\infty}\left(\mathbb{R}_{q,+}\right)$ is continuous at 0 , i.e.,

$$
\lim _{x \rightarrow 0}\left\|T_{x, q}(f)-f\right\|_{\infty, q}=0 .
$$


Proof The result follows from the previous proposition, the properties of the $q$-generalized translation and the Lebesgue theorem.

\section{$3 q$-Bessel Fourier transform}

In [2], we have defined, for $f \in L_{q}^{1}\left(\mathbb{R}_{q,+}, x d_{q} x\right)$, the $q$-Bessel Fourier transform by

$$
\mathcal{F}_{q}(f)(\lambda)=\frac{1}{1-q} \int_{0}^{\infty} f(x) J_{0}\left(\lambda x ; q^{2}\right) x d_{q} x, \quad \lambda \in \widetilde{\mathbb{R}}_{q,+} .
$$

In the following propositions, we summarize some of its properties (see [2]).

\section{Proposition 5}

(1) For $f \in L_{q}^{1}\left(\mathbb{R}_{q,+}, x d_{q} x\right)$, we have

$$
\mathcal{F}_{q}(f) \in \mathcal{C}_{* q, 0}\left(\mathbb{R}_{q,+}\right)
$$

and

$$
\left|\mathcal{F}_{q}(f)(\lambda)\right| \leq \frac{1}{1-q}\|f\|_{1, q}, \quad \lambda \in \widetilde{\mathbb{R}}_{q,+}
$$

(2) For $f \in L_{q}^{1}\left(\mathbb{R}_{q,+}, x d_{q} x\right)$, we have

$$
\mathcal{F}_{q}\left(T_{x, q} f\right)(\lambda)=J_{0}\left(\lambda x ; q^{2}\right) \mathcal{F}_{q}(f)(\lambda), \quad x, \lambda \in \widetilde{\mathbb{R}}_{q,+}
$$

(3) Iff, $D_{q}^{+} f, \Delta_{q} f \in L_{q}^{1}\left(\mathbb{R}_{q,+}, x d_{q} x\right)$ and $x D_{q}^{+} f(x) \rightarrow 0$ as $x \downarrow 0$ in $\mathbb{R}_{q,+}$, then

$$
\mathcal{F}_{q}\left(\Delta_{q} f\right)(\lambda)=-\lambda^{2} \mathcal{F}_{q}(f)(\lambda), \quad \lambda \in \mathbb{R}_{q,+}
$$

Theorem 1 (Plancherel formula) $\mathcal{F}_{q}$ is an isomorphism from $\mathcal{S}_{* q}\left(\mathbb{R}_{q,+}\right)$ onto itself, $\mathcal{F}_{q}^{-1}=$ $\mathcal{F}_{q}$, and for all $f \in \mathcal{S}_{* q}\left(\mathbb{R}_{q,+}\right)$,

$$
\left\|\mathcal{F}_{q}(f)\right\|_{2, q}=\|f\|_{2, q}
$$

Using this result and the relation (37), one can state the following proposition.

Proposition 6 For all $f \in \mathcal{S}_{* q}\left(\mathbb{R}_{q,+}\right)$ and all $x \in \widetilde{\mathbb{R}}_{q,+}$, we have for $\lambda \in \mathbb{R}_{q,+}$,

$$
T_{x, q} f(\lambda)=\frac{1}{1-q} \int_{0}^{\infty} \mathcal{F}_{q}(f)(t) J_{0}\left(t x ; q^{2}\right) J_{0}\left(t \lambda ; q^{2}\right) t d_{q} t
$$

which can be extended for $f \in L^{1}\left(\mathbb{R}_{q,+}, x d_{q} x\right)$.

Proposition 7 For $f \in \mathcal{S}_{* q}\left(\mathbb{R}_{q,+}\right)$ and $y \in \mathbb{R}_{q,+}$, we have

$$
T_{x, q} f(y)=\sum_{n=0}^{+\infty} \frac{q^{n(n+1)}}{\left(q^{2} ; q^{2}\right)_{n}^{2}} y^{2 n}\left(\Delta_{q}^{n} f\right)(x)
$$

where $\Delta_{q}^{0}(f)=f$ and $\Delta_{q}^{n+1}(f)=\Delta_{q}\left[\Delta_{q}^{n}(f)\right], n \in \mathbb{N}$. 
Proof First by the Plancherel formula, we have

$$
f(x)=\frac{1}{1-q} \int_{0}^{\infty} \mathcal{F}_{q}(f)(t) J_{0}\left(t x ; q^{2}\right) t d_{q} t
$$

So,

$$
\Delta_{q}^{n} f(x)=\frac{1}{1-q} \int_{0}^{\infty} \mathcal{F}_{q}(f)(t) \Delta_{q}^{n}\left(J_{0}\left(t x ; q^{2}\right)\right) t d_{q} t=\frac{(-1)^{n}}{1-q} \int_{0}^{\infty} \mathcal{F}_{q}(f)(t) t^{2 n} J_{0}\left(t x ; q^{2}\right) t d_{q} t
$$

On the other hand, from the definition of the function $J_{0}$, the Plancherel formula and the relations (39) and (38), we have

$$
\begin{aligned}
T_{x, q} f(y) & =\frac{1}{1-q} \int_{0}^{\infty} \mathcal{F}_{q}(f)(t) J_{0}\left(t x ; q^{2}\right) \sum_{n=0}^{\infty} \frac{(-1)^{n} q^{n(n+1)} t^{2 n} y^{2 n}}{\left(q^{2} ; q^{2}\right)_{n}^{2}} t d_{q} t \\
& =\sum_{n=0}^{+\infty} \frac{q^{n(n+1)}}{\left(q^{2} ; q^{2}\right)_{n}^{2}} y^{2 n}\left(\Delta_{q}^{n} f\right)(x) .
\end{aligned}
$$

Proposition 8 For $1 \leq p<\infty, \mathcal{S}_{* q}\left(\mathbb{R}_{q,+}\right)$ is dense in $L_{q}^{p}\left(\mathbb{R}_{q,+}, x d_{q} x\right)$.

Proof It suffices to consider functions with compact supports on $\mathbb{R}_{q,+}$.

\section{4 -convolution product}

In [2], the authors defined the $q$-convolution product of two suitable functions as

$$
f *_{B} g(x)=\frac{1}{1-q} \int_{0}^{\infty} T_{x, q} f(y) g(y) y d_{q} y, \quad x \in \mathbb{R}_{q,+} .
$$

It satisfies the following properties (see [2]).

Proposition 9 For $f, g, h \in L_{q}^{1}\left(\mathbb{R}_{q,+}, x d_{q} x\right)$, we have

(1) $f *_{B} g=g *_{B} f$.

(2) $\mathcal{F}_{q}\left(f *_{B} g\right)=\mathcal{F}_{q}(f) \mathcal{F}_{q}(g)$

(3) $\left(f *_{B} g\right) *_{B} h=f *_{B}\left(g *_{B} h\right)$.

In this section, we shall prove that the notion of $q$-convolution product can be extended to functions in $L_{q}^{p}\left(\mathbb{R}_{q,+}, x d_{q} x\right)$ space. We begin by the following result.

Proposition 10 Let $g \in L_{q}^{1}\left(\mathbb{R}_{q,+}, x d_{q} x\right)$ and $f \in L_{q}^{p}\left(\mathbb{R}_{q,+}, x d_{q} x\right), 1 \leq p<\infty$. Then

(1) $\forall x \in \mathbb{R}_{q,+}, y \mapsto T_{x, q}(f)(y) g(y) \in L_{q}^{1}\left(\mathbb{R}_{q,+}, x d_{q} x\right)$.

(2) The function $f *_{B} g \in L_{q}^{p}\left(\mathbb{R}_{q,+}, x d_{q} x\right)$ and

$$
\left\|f *_{B} g\right\|_{p, q} \leq \frac{1}{1-q}\|g\|_{1, q}\|f\|_{p, q} .
$$

Proof

(a) For $p=1$ : 
From Fubini-Tonelli's theorem and Proposition 4, we have

$$
\begin{aligned}
\int_{0}^{\infty}\left|f *_{B} g\right|(x) x d_{q} x & \leq \int_{0}^{\infty}|g(y)|\left(\int_{0}^{\infty}\left|T_{x, q}(f)(y)\right| x d_{q} x\right) y d_{q} y \\
& =\int_{0}^{\infty}|g(y)|\left\|T_{y, q}(f)\right\|_{q, 1} y d_{q} y \leq\|g\|_{1, q}\|f\|_{1, q} .
\end{aligned}
$$

(b) For $1<p<\infty$ :

Let $r \in] 1,+\infty\left[\right.$ be such that $\frac{1}{p}+\frac{1}{r}=1$. For a bounded subset $E$ of $\mathbb{R}_{q,+}$, we note $\chi_{E}$ the characteristic function of $E$.

From Fubini-Tonelli's theorem, we have

$$
\begin{aligned}
\int_{0}^{\infty} & \int_{0}^{\infty}\left|T_{x, q}(f)(y)\right||g(y)| \chi_{E}(x) y d_{q} y x d_{q} x \\
\quad= & \int_{0}^{\infty}|g(y)|\left(\int_{0}^{\infty}\left|T_{x, q}(f)(y)\right| \chi_{E}(x) x d_{q} x\right) y d_{q} y .
\end{aligned}
$$

Using the Holder inequality and Proposition 4, we obtain

$$
\int_{0}^{\infty} \int_{0}^{\infty}\left|T_{x, q}(f)(y)\right||g(y)| \chi_{E}(x) x d_{q} x y d_{q} y \leq\|g\|_{1, q}\|f\|_{p, q}\left(\int_{0}^{\infty} \chi_{E}(x) x d_{q} x\right)^{\frac{1}{r}}<+\infty
$$

Then the function $(x, y) \mapsto T_{x, q}(f)(y) g(y) \chi_{E}(x)$ is integrable on $\mathbb{R}_{q,+} \times \mathbb{R}_{q,+}$ with respect to the measure $x d_{q} x y d_{q} y$. From Fubini's theorem we deduce that for all $x \in E$, the mapping $y \mapsto T_{x, q}(f)(y) g(y)$ belongs to $L_{q}^{1}\left(\mathbb{R}_{q,+}, y d_{q} y\right)$, and the mapping $x \mapsto \chi_{E}(x) \int_{0}^{\infty} T_{x, q}(f)(y) \times$ $g(y) y d_{q} y$ belongs also to $L_{q}^{1}\left(\mathbb{R}_{q,+}, x d_{q} x\right)$. Then the function $x \mapsto \int_{0}^{\infty} T_{x, q}(f)(y) g(y) y d_{q} y$ is measurable.

Furthermore, from the Holder inequality, we have for all $x \in \mathbb{R}_{q,+}$

$$
\begin{aligned}
\left|f *_{B} g(x)\right| & \leq \frac{1}{1-q} \int_{0}^{\infty}\left|T_{x, q}(f)(y)\right||g(y)|^{\frac{1}{p}}|g(y)|^{\frac{1}{r}} y d_{q} y \\
& \leq \frac{1}{1-q}\left(\int_{0}^{\infty}\left|T_{x, q}(f)(y)\right|^{p}|g(y)| y d_{q} y\right)^{\frac{1}{p}}\left(\int_{0}^{\infty}|g(y)| y d_{q} y\right)^{\frac{1}{r}} .
\end{aligned}
$$

Finally, using Fubini's theorem and Proposition 4, we obtain

$$
\left\|f *_{B} g\right\|_{q, p}^{p} \leq\left(\frac{1}{1-q}\right)^{p}\|g\|_{1, q}^{1+\frac{p}{r}}\|f\|_{p, q}^{p}
$$

This completes the proof.

Proposition 11 Let $f$ be in $L_{q}^{p}\left(\mathbb{R}_{q,+}, x d_{q} x\right), 1<p<+\infty$, and $g$ in $L_{q}^{r}\left(\mathbb{R}_{q,+}, x d_{q} x\right), 1<r<+\infty$, such that $\frac{1}{p}+\frac{1}{r}=1$. Then the function $f *_{B} g$ is continuous at 0 , and we have

$$
\sup _{x \in \mathbb{R}_{q,+}}\left|f *_{B} g(x)\right| \leq \frac{1}{1-q}\|f\|_{p, q}\|g\|_{r, q} .
$$


Proof From the Holder inequality and Proposition 4, we have, for $x \in \mathbb{R}_{q,+},\left|f *_{B} g(x)\right| \leq$ $\frac{1}{1-q}\|f\|_{p, q}\|g\|_{r, q}$ and $\left|f *_{B} g(x)-f *_{B} g(0)\right| \leq\left\|T_{x, q}(f)-f\right\|_{p, q}\|g\|_{r, q}$. The continuity of $f *_{B} g$ at 0 follows from Corollary 1.

In the same way, we have the following result.

Proposition 12 Let $f$ be in $L_{q}^{1}\left(\mathbb{R}_{q,+}, x d_{q} x\right)$ and $g$ in $L_{q}^{\infty}\left(\mathbb{R}_{q,+}\right)$. Then the function $f *_{B} g$ is continuous in 0 , bounded and we have

$$
\sup _{x \in \mathbb{R}_{q,+}}\left|f *_{B} g(x)\right| \leq \frac{1}{1-q}\|f\|_{1, q}\|g\|_{\infty, q} .
$$

Proof From the definition of the $q$-convolution product and Proposition 4, we have

$$
\left|f *_{B} g(x)\right| \leq \frac{1}{1-q}\|f\|_{1, q}\|g\|_{\infty, q} .
$$

On the other hand, we have, for $x \in \mathbb{R}_{q,+}$,

$$
\begin{aligned}
\left|f *_{B} g(x)-f *_{B} g(0)\right| & =\frac{1}{1-q}\left|\int_{0}^{+\infty}\left[T_{x, q}(f)(y)-f(y)\right] g(y) y d_{q} y\right| \\
& \leq \frac{1}{1-q}\left\|T_{x, q}(f)-f\right\|_{1, q}\|g\|_{\infty, q},
\end{aligned}
$$

which gives the result by the use of Corollary 1 .

Now, let us adopt the following notation:

For a function $u$ defined on $\mathbb{R}_{q,+}$ and $\alpha \in \mathbb{R}_{q,+}$, we define

$$
u_{\alpha}(x)=\frac{1}{\alpha^{2}} u\left(\frac{x}{\alpha}\right), \quad x \in \mathbb{R}_{q,+} .
$$

Theorem 2 Let $u$ be a non-negative function defined on $\mathbb{R}_{q,+}$ such that

$$
\frac{1}{1-q} \int_{0}^{+\infty} u(x) x d_{q} x=1
$$

Then

(i) For all $f \in L^{p}\left(\mathbb{R}_{q,+}, x d_{q} x\right), 1 \leq p<\infty$, we have

$$
\lim _{n \rightarrow+\infty}\left\|f *_{B} u_{q^{n}}-f\right\|_{p, q}=0
$$

(ii) For all $f \in \mathcal{C}_{* q, 0}\left(\mathbb{R}_{q,+}\right)$, we have

$$
\lim _{n \rightarrow+\infty}\left\|f *_{B} u_{q^{n}}-f\right\|_{\infty, q}=0
$$

Proof (i) From the properties of the $q$-generalized translation, the definition of the $q$-convolution product and the relation (46), we have for all $x \in \mathbb{R}_{q,+}$ and $n \in \mathbb{N}$,

$$
f *_{B} u_{q^{n}}(x)-f(x)=\frac{1}{1-q} \int_{0}^{+\infty} u_{q^{n}}(y)\left[T_{x, q}(f)(y)-f(x)\right] y d_{q} y .
$$


Then

$$
\left|f *_{B} u_{q^{n}}(x)-f(x)\right| \leq \frac{1}{1-q} \int_{0}^{+\infty} u_{q^{n}}(y)\left|T_{x, q}(f)(y)-f(x)\right| y d_{q} y .
$$

So, for $p, r \in] 1,+\infty\left[\right.$ such that $\frac{1}{p}+\frac{1}{r}=1$, we have, by the use of the $q$-Holder inequality and the relation (46),

$$
\begin{aligned}
\left|f *_{B} u_{q^{n}}(x)-f(x)\right|^{p} & \leq\left(\frac{1}{1-q}\right)^{p}\left(\int_{0}^{+\infty} u_{q^{n}}^{\frac{1}{p}}(y) u_{q^{n}}^{\frac{1}{r}}(y)\left|T_{x, q}(f)(y)-f(x)\right| y d_{q} y\right)^{p} \\
& \leq \frac{1}{1-q} \int_{0}^{+\infty} u_{q^{n}}(y)\left|T_{x, q}(f)(y)-f(x)\right|^{p} y d_{q} y .
\end{aligned}
$$

Then

$$
\int_{0}^{+\infty}\left|f *_{B} u_{q^{n}}(x)-f(x)\right|^{p} x d_{q^{\prime}} x \leq \frac{1}{1-q} \int_{0}^{+\infty} \int_{0}^{+\infty} u_{q^{n}}(y)\left|T_{x, q}(f)(y)-f(x)\right|^{p} y d_{q} y x d_{q} x .
$$

The Fubini-Tonelli's theorem leads to

$$
\left\|f *_{B} u_{q^{n}}-f\right\|_{p, q}^{p} \leq \frac{1}{1-q} \int_{0}^{+\infty} u_{q^{n}}(y)\left\|T_{y, q}(f)-f\right\|_{p, q}^{p} y d_{q} y .
$$

The change of variable $t=\frac{y}{q^{n}}$ gives

$$
\left\|f *_{B} u_{q^{n}}-f\right\|_{p, q}^{p} \leq \frac{1}{1-q} \int_{0}^{+\infty} u(t)\left\|T_{q^{n} t, q}(f)-f\right\|_{p, q}^{p} t d_{q} t .
$$

From the dominated convergence theorem, Corollary 1 and Proposition 4, we deduce that

$$
\lim _{n \rightarrow+\infty}\left\|f *_{B} u_{q^{n}}-f\right\|_{p, q}=0 .
$$

(ii) We have, for all $x \in \mathbb{R}_{q,+}$,

$$
\left|f *_{B} u_{q^{n}}(x)-f(x)\right| \leq \frac{1}{1-q} \int_{0}^{+\infty} u_{q^{n}}(y)\left|T_{y, q}(f)(x)-f(x)\right| y d_{q} y .
$$

Thus

$$
\left\|f *_{B} u_{q^{n}}-f\right\|_{\infty, q} \leq \frac{1}{1-q} \int_{0}^{+\infty} u_{q^{n}}(y)\left\|T_{y, q}(f)-f\right\|_{\infty, q} y d_{q} y .
$$

By the change of variables $t=\frac{y}{q^{n}}$, we have

$$
\left\|f *_{B} u_{q^{n}}-f\right\|_{\infty, q} \leq \frac{1}{1-q} \int_{0}^{+\infty} u(t)\left\|T_{q^{n}, q}(f)-f\right\|_{\infty, q} t d_{q} t .
$$

Thus, the dominated convergence theorem, Corollary 1 and Proposition 4 give

$$
\lim _{n \rightarrow+\infty}\left\|f *_{B} u_{q^{n}}-f\right\|_{\infty, q}=0
$$




\section{The $q$-analogue of the heat semi-group}

In this section, we are concerned with the $q$-analogue of the heat semi-group associated with the third Jackson $q$-Bessel function of order zero and we define it on $L_{q}^{1}\left(\mathbb{R}_{q,+}, x d_{q} x\right)$ by the following.

\section{Definition 1}

$$
P_{t, q}(f)(x)=\left(G\left(\cdot, t ; q^{2}\right) *_{B} f\right)(x)=\frac{1}{1-q} \int_{0}^{\infty} T_{x, q} G\left(y, t ; q^{2}\right) f(y) y d_{q} y,
$$

where $t>0, G\left(\cdot, t ; q^{2}\right)$ is the $q$-Gaussian kernel of $P_{t, q}$ defined by

$$
G\left(x, t ; q^{2}\right)=\frac{1}{\left(1-q^{2}\right) t} e_{q^{2}}\left(\frac{-x^{2}}{\left(1-q^{2}\right)^{2} t}\right)
$$

and

$$
e_{q}(x)=\frac{1}{((1-q) x ; q)_{\infty}}, \quad|x|<\frac{1}{1-q} .
$$

Proposition 13 The q-Gaussian kernel satisfies the following properties:

(1)

$$
G\left(x, t ; q^{2}\right)=\mathcal{F}_{q}\left(e_{q^{2}}\left(-t(\cdot)^{2}\right)\right)(x)
$$

(2)

$$
\mathcal{F}_{q}\left(G\left(\cdot, t ; q^{2}\right)\right)(x)=e_{q^{2}}\left(-t x^{2}\right)
$$

Proof (1) On the one hand, we have

$$
\begin{aligned}
\mathcal{F}_{q}\left(e_{q^{2}}\left(-t(\cdot)^{2}\right)\right)(x) & =\frac{1}{1-q} \int_{0}^{\infty} e_{q^{2}}\left(-t y^{2}\right) J_{0}\left(x y ; q^{2}\right) y d_{q} y \\
& =\frac{1}{1-q} \int_{0}^{\infty} e_{q^{2}}\left(-t y^{2}\right) \sum_{k=0}^{\infty} \frac{(-1)^{k} q^{k(k+1)} x^{2 k} y^{2 k}}{\left(q^{2} ; q^{2}\right)_{k}^{2}} y d_{q} y \\
& =\frac{1}{1-q} \sum_{k=0}^{\infty} \frac{(-1)^{k} q^{k(k+1)} x^{2 k}}{\left(q^{2} ; q^{2}\right)_{k}^{2}} \int_{0}^{\infty} e_{q^{2}}\left(-t y^{2}\right) y^{2 k+1} d_{q} y .
\end{aligned}
$$

On the other hand, we have

$$
\begin{aligned}
\int_{0}^{\infty} e_{q^{2}}\left(-t y^{2}\right) y^{2 k+1} d_{q} y & =(1-q) \sum_{n=-\infty}^{\infty} \frac{y^{(2 k+2) n}}{\left(-\left(1-q^{2}\right) t q^{2 n} ; q^{2}\right)_{\infty}} \\
& =\frac{1-q}{\left(-\left(1-q^{2}\right) t ; q^{2}\right)_{\infty}} \sum_{n=-\infty}^{\infty}\left(-\left(1-q^{2}\right) t ; q^{2}\right)_{n}\left(y^{(2 k+2)}\right)^{n}
\end{aligned}
$$

But, by using the Ramanujan identity (see [3]), we obtain

$$
\sum_{n=0}^{\infty}\left(-\left(1-q^{2}\right) t ; q^{2}\right)_{n}\left(y^{(2 k+2)}\right)^{n}=\frac{\left(q^{2},-\left(1-q^{2}\right) t q^{(2 k+2)}, \frac{q^{-2 k}}{-\left(1-q^{2}\right) t} ; q^{2}\right)_{\infty}}{\left(\frac{q^{2}}{-\left(1-q^{2}\right) t}, q^{2 k+2} ; q^{2}\right)_{\infty}} .
$$


This gives

$$
\int_{0}^{\infty} e_{q^{2}}\left(-t y^{2}\right) y^{2 k+1} d_{q} y=(1-q)\left(q^{2} ; q^{2}\right)_{k}\left(\frac{1}{\left(1-q^{2}\right) t}\right)^{k+1} q^{-k^{2}-k}
$$

Thus

$$
\begin{aligned}
\mathcal{F}_{q}\left(e_{q^{2}}\left(-t y^{2}\right)\right)(x) & =\sum_{k=0}^{\infty} \frac{(-1)^{k} x^{2 k}}{\left(q^{2} ; q^{2}\right)_{k}}\left(\frac{1}{\left(1-q^{2}\right) t}\right)^{k+1} \\
& =\frac{1}{\left(1-q^{2}\right) t} e_{q^{2}}\left(\frac{-x^{2}}{\left(1-q^{2}\right)^{2} t}\right) \\
& =G\left(x, t ; q^{2}\right) .
\end{aligned}
$$

(2) By the use of Plancherel formula and Theorem 1, we obtain

$$
\mathcal{F}_{q}\left(G\left(\cdot, t ; q^{2}\right)\right)(x)=e_{q^{2}}\left(-t x^{2}\right) .
$$

Using this second equality and equalizing terms by terms, we obtain the following lemma.

\section{Lemma 2}

$$
\int_{0}^{\infty} G\left(\cdot, t ; q^{2}\right)(y) y^{2 n+1} d_{q} y=(1-q)\left(q^{2} ; q^{2}\right)_{n}\left(1-q^{2}\right)^{n} t^{n} q^{-n^{2}-n} .
$$

Now, we are in a situation to state some properties of the heat semi-group $P_{t, q}$.

Proposition 14 The following properties hold:

(1) For all $\in \in \mathcal{S}_{* q}\left(\mathbb{R}_{q,+}\right)$,

$$
P_{t, q}(f)(x)=e_{q^{2}}\left(t \Delta_{q} f\right)(x)
$$

(2) For all $f \in \mathcal{S}_{* q}\left(\mathbb{R}_{q,+}\right)$ such that $f \geq 0$ and $t>0$, we have

$$
P_{t, q} f \geq 0 \text {. }
$$

Proof (1) From the definition of $P_{t, q}$, the properties of the $q$-generalized translation and Lemma 2, we have

$$
\begin{aligned}
P_{t, q}(f)(x) & =\frac{1}{1-q} \int_{0}^{\infty} T_{x, q} G\left(\cdot, t ; q^{2}\right)(y) f(y) y d_{q} y=\frac{1}{1-q} \int_{0}^{\infty} G\left(\cdot, t ; q^{2}\right)(y) T_{x, q} f(y) y d_{q} y \\
& =\frac{1}{1-q} \sum_{n=0}^{+\infty} \frac{q^{n^{2}+n}}{\left(q^{2} ; q^{2}\right)_{n}^{2}}\left(\int_{0}^{\infty} G\left(\cdot, t ; q^{2}\right)(y) y^{2 n+1} d_{q} y\right)\left(\Delta_{q}^{n} f\right)(x) \\
& =\sum_{n=0}^{+\infty} \frac{t^{n}\left(1-q^{2}\right)^{n} \Delta_{q}^{n} f(x)}{\left(q^{2} ; q^{2}\right)_{n}}=e_{q^{2}}\left(t \Delta_{q} f\right)(x) .
\end{aligned}
$$

(2) Follows from the positivity of the $q$-generalized translation and the fact that $G\left(x, t ; q^{2}\right) \geq 0$. 
Since $G\left(\cdot, t ; q^{2}\right) \in \mathcal{S}_{* q}\left(\mathbb{R}_{q,+}\right)$, then Proposition 10 implies that $P_{q, t}$ can be extended to $L_{q}^{p}\left(\mathbb{R}_{q,+}, x d_{q} x\right), 1 \leq p<\infty$ and we have the following.

Proposition 15 For all $f \in L_{q}^{p}\left(\mathbb{R}_{q,+}, x d_{q} x\right), 1 \leq p<\infty, P_{q, t} f \in L_{q}^{p}\left(\mathbb{R}_{q,+}, x d_{q} x\right)$ and

$$
\left\|P_{q, t} f\right\|_{p, q} \leq\|f\|_{p, q} .
$$

Proof Using Lemma 2, we have

$$
\left\|G\left(\cdot, t ; q^{2}\right)\right\|_{1, q}=\int_{0}^{\infty} G\left(y, t ; q^{2}\right) y d_{q} y=(1-q) .
$$

So, the result follows by using this equality and Proposition 10.

Now, for $f(x)=\sum_{n=0}^{\infty} a_{n} x^{n}, t, t^{\prime} \in \mathbb{R}$, we note

$$
f\left(\left[t+t^{\prime}\right]_{q}\right)=\sum_{n=0}^{\infty} a_{n}\left[t+t^{\prime}\right]_{q}^{n}
$$

where $\left[t+t^{\prime}\right]_{q}^{n}=\left(t+t^{\prime}\right)\left(t+q t^{\prime}\right) \cdots\left(t+q^{n-1} t^{\prime}\right)$ if $n \neq 0$ and $\left[t+t^{\prime}\right]_{q}^{0}=1$ (see [9]).

It is clear that $f(t)=f\left([t+0]_{q}\right)$ and we have

$$
\lim _{q \rightarrow 1}\left[t+t^{\prime}\right]_{q}^{n}=\left(t+t^{\prime}\right)^{n}
$$

On the other hand, we have (see [10])

$$
e_{q^{2}}\left(\left[t+t^{\prime}\right]_{q^{2}}\right)=\frac{e_{q^{2}}(t)}{e_{q^{2}}\left(-t^{\prime}\right)}
$$

Using this equality and Proposition 14, one can state the following result.

Proposition 16 For $f \in \mathcal{S}_{* q}\left(\mathbb{R}_{q,+}\right)$, and $t>-t^{\prime}>0$, we have

$$
P_{-t^{\prime}, q}(f) \cdot P_{\left[t+t^{\prime}\right]_{q^{2}, q}}(f)=P_{t, q}(f) .
$$

\section{Remarks}

(1) From the relation (59) and the fact that $e_{q^{2}}$ is a $q$-analogue of the classical exponential function, we can see that $\left(P_{t, q}\right)_{t>0}$ as a $q$-analogue of the classical heat semi-group.

(2) For two formal $q^{2}$-commuting variables $t$ and $t^{\prime}\left(t t^{\prime}=q^{2} t^{\prime} t\right)$, we have

$$
P_{t+t^{\prime}, q}(f)=P_{t, q}(f) P_{t^{\prime}, q}(f),
$$

which proves that $P_{t, q}$ is a $q$-analogue of the classical heat semi-group. 


\section{The $q$-analogue of the Wiener algebra}

In this section, we attempt to study the following $q$-analogue of the Wiener algebra:

$$
\mathbf{A}\left(\mathbb{R}_{q,+}\right)=\left\{f \in L_{q}^{1}\left(\mathbb{R}_{q,+}, x d_{q} x\right) ; \mathcal{F}_{q}(f) \in L_{q}^{1}\left(\mathbb{R}_{q,+}, x d_{q} x\right)\right\}
$$

Note that

$$
\mathcal{S}_{* q}\left(\mathbb{R}_{q,+}\right) \subset \mathbf{A}\left(\mathbb{R}_{q,+}\right) \subset L_{q}^{1}\left(\mathbb{R}_{q,+}\right) .
$$

We begin by the following results which are useful in the sequel.

Proposition 17 For all function $\mathcal{C}_{* q, b}\left(\widetilde{\mathbb{R}}_{q,+}\right)$, we have

$$
\lim _{n \rightarrow+\infty} \frac{1}{1-q} \int_{0}^{+\infty} f(x) G_{q^{n}}\left(\cdot, t ; q^{2}\right)(x) x d_{q} x=f(0),
$$

where $G_{q^{n}}\left(\cdot, t ; q^{2}\right)$ is defined in $(45)$.

Proof It follows from the fact that

$$
\frac{1}{1-q} \int_{0}^{+\infty} G\left(x, t ; q^{2}\right) x d_{q} x=1
$$

and the following lemma.

Lemma 3 Let $u \in L_{q}^{1}\left(\mathbb{R}_{q,+}, x d_{q} x\right)$ be such that $\int_{0}^{+\infty} u(x) x d_{q} x=1$, then for all function $f \in$ $\mathcal{C}_{* q, b}\left(\widetilde{\mathbb{R}}_{q,+}\right)$, we have

$$
\lim _{n \rightarrow+\infty} \int_{0}^{+\infty} f(x) u_{q^{n}}(x) x d_{q^{x}} x=f(0) .
$$

Proof $[($ of the lemma $)]$

$$
\begin{aligned}
\left|\int_{0}^{+\infty} f(x) u_{q^{n}}(x) x d_{q} x-f(0)\right| & =\left|\int_{0}^{+\infty} f(x) u_{q^{n}}(x) x d_{q} x-\int_{0}^{+\infty} f(0) u(x) x d_{q} x\right| \\
& =\left|\int_{0}^{+\infty} f(x) \frac{1}{q^{2 n}} u\left(\frac{x}{q^{n}}\right) x d_{q^{x}} x-\int_{0}^{+\infty} f(0) u(x) x d_{q^{\prime}} x\right| \\
& =\left|\int_{0}^{+\infty}\left(f\left(q^{n} x\right)-f(0)\right) u(x) x d_{q} x\right| \\
& \leq \int_{0}^{+\infty}\left|f\left(q^{n} x\right)-f(0)\right||u(x)| x d_{q} x .
\end{aligned}
$$

Finally, the dominated convergence theorem achieves the proof.

Theorem 3 Let $f \in L_{q}^{p}\left(\mathbb{R}_{q,+}, x d_{q} x\right), 1 \leq p<\infty$, then we have

$$
\lim _{n \rightarrow+\infty}\left\|f-f *_{B} G_{q^{n}}\left(\cdot, t, q^{2}\right)\right\|_{p, q}=0 .
$$


Proof The result follows from Theorem 2 and the fact that

$$
\frac{1}{1-q} \int_{0}^{+\infty} G\left(x, t ; q^{2}\right) x d_{q} x=1
$$

Theorem 4 For $f \in L_{q}^{1}\left(\mathbb{R}_{q,+}, x d_{q} x\right)$ such that $\mathcal{F}_{q}(f) \in L_{q}^{1}\left(\mathbb{R}_{q,+}, x d_{q} x\right)$, then

$$
f(x)=\frac{1}{1-q} \int_{0}^{\infty} \mathcal{F}_{q}(f)(y) J_{0}\left(x y ; q^{2}\right) y d_{q} y .
$$

Proof Using the inequality

$$
(1-q) x^{2}\left|f(x)-f *_{B} G_{q^{n}}\left(\cdot, t, q^{2}\right)(x)\right| \leq\left\|f-f *_{B} G_{q^{n}}\left(\cdot, t, q^{2}\right)\right\|_{1, q^{\prime}} \quad \forall x \in \mathbb{R}_{q,+},
$$

and Theorem 3 , we have

$$
f(x)=\lim _{n \rightarrow+\infty} f *_{B} G_{q^{n}}\left(\cdot, t, q^{2}\right)(x) .
$$

Furthermore, using the fact that $f *_{B} G_{q^{n}}\left(\cdot, t, q^{2}\right) \in \mathcal{S}_{* q}\left(\mathbb{R}_{q,+}\right)$ and the Plancherel formula, we have

$$
\begin{aligned}
f *_{B} G_{q^{n}}\left(\cdot, t, q^{2}\right)(x) & =\mathcal{F}_{q}^{-1} \mathcal{F}_{q}\left(f *_{B} G_{q^{n}}\left(\cdot, t, q^{2}\right)\right)(x)=\mathcal{F}_{q}\left(\mathcal{F}_{q}(f) \mathcal{F}_{q}\left(G_{q^{n}}\left(\cdot, t, q^{2}\right)\right)\right)(x) \\
& =\frac{1}{1-q} \int_{0}^{\infty} \mathcal{F}_{q}(f)(y) \mathcal{F}_{q}\left(G_{q^{n}}\left(\cdot, t, q^{2}\right)\right)(y) J_{0}\left(x y ; q^{2}\right) y d_{q} y .
\end{aligned}
$$

On the other hand, we have

$$
\begin{aligned}
\mathcal{F}_{q}\left(G_{q^{n}}\left(\cdot, t, q^{2}\right)\right)(y) & =\frac{1}{1-q} \int_{0}^{\infty} G_{q^{n}}\left(\cdot, t, q^{2}\right)(u) J_{0}\left(u y ; q^{2}\right) u d_{q} u \\
& =\frac{1}{q^{2 n}(1-q)} \int_{0}^{\infty} G\left(\frac{u}{q^{n}}, t, q^{2}\right) J_{0}\left(u y ; q^{2}\right) u d_{q} u \\
& =\frac{1}{1-q} \int_{0}^{\infty} G\left(u, t ; q^{2}\right) J_{0}\left(q^{n} u y ; q^{2}\right) u d_{q} u \\
& =\mathcal{F}_{q}\left(G\left(\cdot, t ; q^{2}\right)\right)\left(q^{n} y\right) \\
& =e_{q^{2}}\left(-t q^{2 n} y^{2}\right) .
\end{aligned}
$$

This gives

$$
f *_{B} G_{q^{n}}\left(\cdot, t, q^{2}\right)(x)=\frac{1}{1-q} \int_{0}^{\infty} \mathcal{F}_{q}(f)(y) e_{q^{2}}\left(-t q^{2 n} y^{2}\right) J_{0}\left(x y ; q^{2}\right) y d_{q} y .
$$

Thus, the dominated convergence theorem leads to

$$
\begin{aligned}
f(x) & =\frac{1}{1-q} \lim _{n \rightarrow+\infty} \int_{0}^{\infty} \mathcal{F}_{q}(f)(y) e_{q^{2}}\left(-t q^{2 n} y^{2}\right) J_{0}\left(x y ; q^{2}\right) y d_{q} y \\
& =\frac{1}{1-q} \int_{0}^{\infty} \mathcal{F}_{q}(f)(y) J_{0}\left(x y ; q^{2}\right) y d_{q} y .
\end{aligned}
$$

In the following result, we summarize some of its density properties. 
Proposition 18 We have

(1)

$$
\mathbf{A}\left(\mathbb{R}_{q,+}\right) \subset L_{q}^{p}\left(\mathbb{R}_{q,+}, x d_{q} x\right) \text { and } \overline{\mathbf{A}\left(\mathbb{R}_{q,+}\right)}=L_{q}^{p}\left(\mathbb{R}_{q,+}, x d_{q} x\right)
$$

(2)

$$
\mathbf{A}\left(\mathbb{R}_{q,+}\right) \subset \mathcal{C}_{* q, 0}\left(\mathbb{R}_{q,+}\right) \text { and } \overline{\mathbf{A}\left(\mathbb{R}_{q,+}\right)}=\mathcal{C}_{* q, 0}\left(\mathbb{R}_{q,+}\right)
$$

Proof Let $f \in \mathbf{A}\left(\mathbb{R}_{q,+}\right)$, then $f \in L_{q}^{1}\left(\mathbb{R}_{q,+}, x d_{q} x\right)$ and $\mathcal{F}_{q}(f) \in L_{q}^{1}\left(\mathbb{R}_{q,+}, x d_{q} x\right)$.

By Theorem 4 and the fact that $\left|J_{0}\left(x ; q^{2}\right)\right| \leq 1$ (see[2]), we have $|f| \leq \frac{1}{1-q}\left\|\mathcal{F}_{q}(f)\right\|_{1, q}$ and

$$
\|f\|_{p, q}^{p} \leq\left(\frac{1}{1-q}\left\|\mathcal{F}_{q}(f)\right\|_{1, q}\right)^{p-1}\|f\|_{1, q} .
$$

Thus, $\mathbf{A}\left(\mathbb{R}_{q,+}\right) \subset L_{q}^{p}\left(\mathbb{R}_{q,+}, x d_{q} x\right)$.

On the other hand, we have by Theorem $4, f=\mathcal{F}_{q}\left(\mathcal{F}_{q}(f)\right)$ and $\mathcal{F}_{q}(f) \in L_{q}^{1}\left(\mathbb{R}_{q,+}, x d_{q} x\right)$. Then, by Proposition $5, f \in \mathcal{C}_{* q, 0}\left(\mathbb{R}_{q,+}\right)$. Thus

$$
\mathbf{A}\left(\mathbb{R}_{q,+}\right) \subset \mathcal{C}_{* q, 0}\left(\mathbb{R}_{q,+}\right)
$$

(1) Let $f \in L_{q}^{p}\left(\mathbb{R}_{q,+}, x d_{q} x\right)$. For $\varepsilon>0$, there exists an $h_{\varepsilon}=h \in L_{q}^{p}\left(\mathbb{R}_{q,+}, x d_{q} x\right)$ with compact support in $\left[q^{k}, q^{-k}\right]$ such that $\|f-h\|_{p, q}<\varepsilon$. By using Theorem 3 , we have

$$
\lim _{n \rightarrow+\infty}\left\|h-h *_{B} G_{q^{n}}\left(\cdot, t, q^{2}\right)\right\|_{p, q}=0 .
$$

Let us show that $h_{n}=h *_{B} G_{q^{n}} \in \mathbf{A}\left(\mathbb{R}_{q,+}\right)$. We have

$$
\left\|h_{n}\right\|_{1, q} \leq \frac{1}{1-q}\|h\|_{1, q}\left\|G_{q^{n}}\left(\cdot, t, q^{2}\right)\right\|_{1, q}
$$

which gives $h_{n} \in L_{q}^{1}\left(\mathbb{R}_{q,+}, x d_{q} x\right)$. Furthermore, we have

$$
\left|\mathcal{F}_{q}\left(h_{n}\right)(t)\right|=\left|\mathcal{F}_{q}(h)(t) e_{q^{2}}\left(-t q^{2 n} u^{2}\right)\right| \leq C e_{q^{2}}\left(-t q^{2 n} u^{2}\right)
$$

with $C$ is some constant.

Since $t \mapsto e_{q^{2}}\left(-t q^{2 n} u^{2}\right)$ belongs to $L_{q}^{1}\left(\mathbb{R}_{q,+}, d_{q} x\right)$ (see [11] ), we have $\mathcal{F}_{q}\left(h_{n}\right) \in L_{q}^{1}\left(\mathbb{R}_{q,+}\right.$, $\left.x d_{q} x\right)$.

(2) Let $f \in \mathcal{C}_{* q, 0}\left(\mathbb{R}_{q,+}\right)$. For $\varepsilon>0$, there exists an $h_{\varepsilon}=h \in L_{q}^{p}\left(\mathbb{R}_{q,+}, x d_{q} x\right)$ with compact support in $\left[q^{k}, q^{-k}\right]$ such that $\|f-h\|_{\infty, q}<\varepsilon$.

Using the inequality

$$
\left((1-q) x^{2}\right)^{\frac{1}{p}}\left|h(x)-h_{n}(x)\right| \leq\left\|h-h_{n}\right\|_{p, q}
$$

and (1), we obtain

$$
\lim _{n \rightarrow+\infty} \sup _{x \geq q^{k}}\left|h(x)-h_{n}(x)\right|=0 .
$$


Now, for all $x<q^{k}$, we have

$$
h_{n}(x)-h(x)=h_{n}(x)=\sum_{i=-k}^{k} q^{2 i} h\left(q^{i}\right) T_{x, q} G_{q^{n}}\left(\cdot, t, q^{2}\right)\left(q^{i}\right) \text {. }
$$

On the other hand, we have

$$
\begin{aligned}
T_{x, q} G_{q^{n}}\left(\cdot, t, q^{2}\right)\left(q^{i}\right) & =\frac{1}{1-q} \int_{0}^{\infty} \mathcal{F}_{q}\left(G_{q^{n}}\left(\cdot, t, q^{2}\right)\right)(u) J_{0}\left(u x ; q^{2}\right) J_{0}\left(u q^{i} ; q^{2}\right) u d_{q} u \\
& =\frac{1}{1-q} \int_{0}^{\infty} e_{q^{2}}\left(-t q^{2 n} u^{2}\right) J_{0}\left(u x ; q^{2}\right) J_{0}\left(u q^{i} ; q^{2}\right) u d_{q} u .
\end{aligned}
$$

Therefore, there exists $k_{n} \geq k+1$ such that

$$
\sup _{x<q^{k}} T_{x, q} G_{q^{n}}\left(\cdot, t, q^{2}\right)\left(q^{i}\right)=T_{q^{k n}, q} G_{q^{n}}\left(\cdot, t, q^{2}\right)\left(q^{i}\right) .
$$

$q^{k_{n}} \in\left\{q^{j}\right\}_{j \geq k+1} \cup\{0\}$ is a compact subset in $\widetilde{\mathbb{R}}_{q,+}$, then there exists a convergent subsequence $\left(q^{\varphi(n)}\right)$ of $\left(q^{k_{n}}\right)$.

- If $\left(q^{\varphi(n)}\right)$ tends to $q^{l}$, then

$$
\begin{aligned}
\lim _{n \rightarrow+\infty} T_{q^{\varphi(n)}, q} G_{q^{n}}\left(\cdot, t, q^{2}\right)\left(q^{i}\right) & =\frac{1}{1-q} \int_{0}^{\infty} J_{0}\left(u q^{l} ; q^{2}\right) J_{0}\left(u q^{i} ; q^{2}\right) u d_{q} u \\
& =\frac{1}{q^{l+i}} \sum_{j=-\infty}^{+\infty} q^{l+i} J_{0}\left(q^{l+j} ; q^{2}\right) J_{0}\left(q^{i+j} ; q^{2}\right) q^{2 j}=0 .
\end{aligned}
$$

- If $\left(q^{\varphi(n)}\right)$ tends to 0 , then

$$
\begin{aligned}
\lim _{n \rightarrow+\infty} T_{q^{\varphi(n)}, q} G_{q^{n}}\left(\cdot, t, q^{2}\right)\left(q^{i}\right) & =\frac{1}{1-q} \int_{0}^{\infty} J_{0}\left(u q^{i} ; q^{2}\right) u d_{q} u \\
& =\frac{1}{1-q} \lim _{b \rightarrow+\infty} \int_{0}^{b} J_{0}\left(u q^{i} ; q^{2}\right) u d_{q} u \\
& =-\frac{1}{1-q} \lim _{b \rightarrow+\infty} \int_{0}^{b} \frac{1}{q^{2 i}} \Delta_{q}\left(J_{0}\left(u q^{i} ; q^{2}\right)\right) u d_{q} u \\
& =-\frac{1-q}{q^{2 i}} \lim _{b \rightarrow+\infty} \int_{0}^{b} D_{q}\left[u D_{q}^{+}\left(J_{0}\left(u q^{i} ; q^{2}\right)\right)\right] d_{q} u \\
& =-\frac{1-q}{q^{2 i}} \lim _{b \rightarrow+\infty}\left[u D_{q}^{+}\left(J_{0}\left(u q^{i} ; q^{2}\right)\right)\right]_{0}^{b} \\
& =-\frac{1}{q^{2 i}} \lim _{b \rightarrow+\infty}\left(J_{0}\left(b q^{i-1} ; q^{2}\right)-J_{0}\left(b q^{i} ; q^{2}\right)\right)=0 .
\end{aligned}
$$

This gives

$$
\lim _{n \rightarrow \infty} \sup _{x \in \mathbb{R}_{q,+}}\left|h(x)-h_{\varphi(n)}(x)\right|=0 .
$$

Theorem 5 For $f \in L_{q}^{2}\left(\mathbb{R}_{q,+}, x d_{q} x\right)$, then

$$
\left\|\mathcal{F}_{q}(f)\right\|_{2, q}=\|f\|_{2, q} .
$$


Proof For $f \in L_{q}^{2}\left(\mathbb{R}_{q,+}, x d_{q} x\right)$ and $f_{n}=f *_{B} G_{q^{n}\left(\cdot, t, q^{2}\right)}$, we have

$$
\mathcal{F}_{q}\left(f_{n}\right)(u)=e_{q^{2}}\left(-t q^{2 n} u^{2}\right) \mathcal{F}_{q}(f)(u)
$$

On the other hand, using the fact that $f_{n} \in \mathcal{S}_{* q}\left(\mathbb{R}_{q,+}\right)$, Theorem 4 gives

$$
f_{n}(x)=\frac{1}{1-q} \int_{0}^{\infty} \mathcal{F}_{q}\left(f_{n}\right)(u) J_{0}\left(x u ; q^{2}\right) u d_{q} u,
$$

which gives

$$
\begin{aligned}
\int_{0}^{\infty} f(x) f_{n}(x) x d_{q} x & =\int_{0}^{\infty} \mathcal{F}_{q}\left(f_{n}\right)(x) \mathcal{F}_{q}(f)(x) x d_{q^{x}} \\
& =\int_{0}^{\infty}\left(\mathcal{F}_{q}(f)(x)\right)^{2} e_{q^{2}}\left(-t q^{2 n} x^{2}\right) x d_{q} x
\end{aligned}
$$

By using Theorem 2, we obtain

$$
\lim _{n \rightarrow+\infty} \int_{0}^{\infty}\left(\mathcal{F}_{q}(f)(x)\right)^{2} e_{q^{2}}\left(-t q^{2 n} x^{2}\right) x d_{q} x=\|f\|_{2, q}^{2}
$$

Since the sequence $e_{q^{2}}\left(-t q^{2 n} x^{2}\right)$ is increasing, the use of Fatou Beppo-Levi theorem achieves the result.

Theorem 6 Let $1<p \leq 2$ and $\frac{1}{p}+\frac{1}{p^{\prime}}=1$. If $\in \in L_{q}^{p}\left(\mathbb{R}_{q,+}, x d_{q} x\right)$, then $\mathcal{F}_{q}(f) \in L_{q}^{p^{\prime}}\left(\mathbb{R}_{q,+}, x d_{q} x\right)$ and

$$
\left\|\mathcal{F}_{q}(f)\right\|_{p^{\prime}, q} \leq B_{p, q}\|f\|_{p, q}
$$

where

$$
B_{p, q}=\left(\frac{1}{1-q}\right)^{\left(-1+\frac{2}{p}\right)} \text {. }
$$

Proof We have, by Theorem 5,

$$
\mathcal{F}_{q}: L_{q}^{2}\left(\mathbb{R}_{q,+}, x d_{q} x\right) \rightarrow L_{q}^{2}\left(\mathbb{R}_{q,+}, x d_{q} x\right)
$$

is a linear isomorphism with norm 1.

On the other hand, we have

$$
\left|\mathcal{F}_{q}(f)(\lambda)\right| \leq \frac{1}{1-q} \int_{0}^{\infty}|f(x)| x d_{q} x, \quad \lambda \in \mathbb{R}_{q,+},
$$

which gives

$$
\left\|\mathcal{F}_{q}(f)\right\|_{\infty, q} \leq \frac{1}{1-q}\|f\|_{1, q} .
$$


So,

$$
\mathcal{F}_{q}: L_{q}^{1}\left(\mathbb{R}_{q,+}, x d_{q} x\right) \rightarrow L_{q}^{\infty}\left(\mathbb{R}_{q,+}, x d_{q} x\right)
$$

is with norm bounded by $\frac{1}{1-q}$.

Finally, the use of the Riesz-Thorin theorem gives the result (see [12]).

Proposition 19 Let $1<p, p^{\prime}, r \leq 2$ be such that $\frac{1}{p}+\frac{1}{p^{\prime}}-1=\frac{1}{r}$ and $\frac{1}{r}+\frac{1}{r^{\prime}}=1$. If $f \in$ $L_{q}^{p}\left(\mathbb{R}_{q,+}, x d_{q} x\right)$ and $g \in L_{q}^{p^{\prime}}\left(\mathbb{R}_{q,+}, x d_{q} x\right)$, then $f *_{B} g \in L_{q}^{r}\left(\mathbb{R}_{q,+}, x d_{q} x\right)$

$$
f *_{B} g=\mathcal{F}_{q}\left(\mathcal{F}_{q}(f) \mathcal{F}_{q}(g)\right)
$$

and

$$
\left\|f *_{B} g\right\|_{r, q} \leq B_{p, q} B_{p^{\prime}, q} B_{r^{\prime}, q}\|f\|_{p, q}\|g\|_{p^{\prime}, q},
$$

where $B_{p, q}, B_{p^{\prime}, q}$ and $B_{r^{\prime}, q}$ are defined by $(72)$.

Proof (i) If $f$ and $g$ belong to $\mathcal{S}_{* q}\left(\mathbb{R}_{q,+}\right)$ the result is clear.

(ii) If $f \in L_{q}^{p}\left(\mathbb{R}_{q,+}, x d_{q} x\right)$ and $g \in L_{q}^{p^{\prime}}\left(\mathbb{R}_{q,+}, x d_{q} x\right)$, we consider two sequences $\left(f_{n}\right)_{n \geq 0}$ and $\left(g_{n}\right)_{n \geq 0}$ in $\mathcal{S}_{* q}\left(\mathbb{R}_{q,+}\right)$ which converge to $f$ and $g$ respectively in $L_{q}^{p}\left(\mathbb{R}_{q,+}, x d_{q} x\right)$ and $L_{q}^{p^{\prime}}\left(\mathbb{R}_{q,+}\right)$. From i) we have $f_{n} *_{B} g_{n}=\mathcal{F}_{q}\left(\mathcal{F}_{q}\left(f_{n}\right) \mathcal{F}_{q}\left(g_{n}\right)\right)$.

We have $\frac{1}{p_{1}}+\frac{1}{p_{1}^{\prime}}=1$ with $p_{1}=\frac{p}{p-1}$ and $p_{1}^{\prime}=\frac{p^{\prime}}{p^{\prime}-1}$ the dual exponents of $p$ and $p^{\prime}$ respectively, which belong to $[2,+\infty[$. From Theorem 6 and the Holder inequality, we have

$$
\begin{aligned}
& \left\|\mathcal{F}_{q}\left(f_{n}\right) \mathcal{F}_{q}\left(g_{n}\right)-\mathcal{F}_{q}(f) \mathcal{F}_{q}(g)\right\|_{r^{\prime}, q} \\
& \leq\left\|\mathcal{F}_{q}(f)\right\|_{p_{1}, q}\left\|\mathcal{F}_{q}\left(g_{n}\right)-\mathcal{F}_{q}(g)\right\|_{p_{1}^{\prime}, q} \\
& \quad+\left\|\mathcal{F}_{q}(g)\right\|_{p_{1}^{\prime}, q}\left\|\mathcal{F}_{q}\left(f_{n}\right)-\mathcal{F}_{q}(f)\right\|_{p_{1}, q} \\
& \quad+\left\|\mathcal{F}_{q}\left(f_{n}\right)-\mathcal{F}_{q}(f)\right\|_{p_{1}, q}\left\|\mathcal{F}_{q}\left(g_{n}\right)-\mathcal{F}_{q}(g)\right\|_{p^{\prime}, q^{\prime}}
\end{aligned}
$$

We deduce that the sequence $\left(\mathcal{F}_{q}\left(f_{n}\right) \mathcal{F}_{q}\left(g_{n}\right)\right)_{n \geq 0}$ converges to $\mathcal{F}_{q}(f) \mathcal{F}_{q}(g)$ in $L_{q}^{r^{\prime}}\left(\mathbb{R}_{q,+}, x d_{q} x\right)$. Theorem 6 implies that the sequence $\left(\mathcal{F}_{q}\left(\mathcal{F}_{q}\left(f_{n}\right) \mathcal{F}_{q}\left(g_{n}\right)\right)\right)_{n \geq 0}$ converges to $\mathcal{F}_{q}\left(\mathcal{F}_{q}(f) \mathcal{F}_{q}(g)\right)$ in $L_{q}^{r}\left(\mathbb{R}_{q,+}, x d_{q} x\right)$.

On the other hand, from Propositions 9 and 10, we have

$$
\begin{aligned}
\left\|f_{n} *_{B} g_{n}-f *_{B} g\right\|_{r, q} \leq & \frac{1}{1-q}\|f\|_{p, q}\left\|g_{n}-g\right\|_{p^{\prime}, q}+\frac{1}{1-q}\|g\|_{p^{\prime}, q}\left\|f_{n}-f\right\|_{p, q} \\
& +\frac{1}{1-q}\left\|f_{n}-f\right\|_{p, q}\left\|g_{n}-g\right\|_{p^{\prime}, q} .
\end{aligned}
$$

Thus, the sequence $\left(f_{n} *_{B} g_{n}\right)_{n \geq 0}$ converges to $f *_{B} g$ in $L_{q}^{r}\left(\mathbb{R}_{q,+}, x d_{q} x\right)$. And so

$$
f *_{B} g=\mathcal{F}_{q}\left(\mathcal{F}_{q}(f) \mathcal{F}_{q}(g)\right) \text {. }
$$


To prove the inequality, we have, by the use of Theorem 6,

$$
\begin{aligned}
\left\|f *_{B} g\right\|_{r, q} \leq\left\|\mathcal{F}_{q}\left(\mathcal{F}_{q}(f) \mathcal{F}_{q}(g)\right)\right\|_{r, q} & \leq B_{r^{\prime}, q}\left\|\mathcal{F}_{q}(f) \mathcal{F}_{q}(g)\right\|_{r^{\prime}, q} \\
& \leq B_{r^{\prime}, q}\left\|\mathcal{F}_{q}(f)\right\|_{p_{1}, q}\left\|\mathcal{F}_{q}(g)\right\|_{p^{\prime}, q^{\prime}}
\end{aligned}
$$

Thus

$$
\left\|f *_{B} g\right\|_{r, q} \leq B_{p, q} B_{p^{\prime}, q} B_{r^{\prime}, q}\|f\|_{p, q}\|g\|_{p^{\prime}, q} .
$$

From the last proposition we deduce the hypercontractivity of the $q$-analogue of the heat semi-group $P_{t, q}$.

Theorem 7 Let $f \in L_{q}^{1}\left(\mathbb{R}_{q,+}, x d_{q} x\right)$ and $t \in \mathbb{R}_{q^{2},+}$. Then

$$
\left\|P_{t, q}\right\|_{r, q} \leq B_{p, q} B_{r^{\prime}, q} t^{-\frac{1}{2 p_{1}}} \alpha\left(p_{1}, q\right)\|f\|_{p, q}
$$

where $\frac{1}{p}-\frac{1}{p_{1}}=\frac{1}{r}, \frac{1}{r}+\frac{1}{r^{\prime}}=1, P_{t, q}$ is given by $(50)$ and $\alpha\left(p_{1}, q\right)=\left\|e_{q^{2}}\left(-(\cdot)^{2}\right)\right\|_{p_{1}, q}$.

Proof By Theorem (6) we have

$$
\left\|P_{q, t}\right\|_{r, q} \leq B_{p, q} B_{p^{\prime}, q} B_{r^{\prime}, q}\|f\|_{p, q}\left\|G_{q^{n}\left(\cdot, t, q^{2}\right)}\right\|_{p^{\prime}, q},
$$

where $\frac{1}{p^{\prime}}+\frac{1}{p_{1}}=1$.

By Theorem (1) and (53), we have

$$
\begin{aligned}
\left\|G_{q^{n}}\left(\cdot, t, q^{2}\right)\right\|_{p^{\prime}, q} & =\left\|\mathcal{F}_{q}\left(\mathcal{F}_{q}\left(G_{q^{n}}\left(\cdot, t, q^{2}\right)\right)\right)\right\|_{p^{\prime}, q} \\
& \leq B_{p_{1}, q}\left\|e_{q^{2}}(-t(\cdot))^{2}\right\|_{p_{1}, q} .
\end{aligned}
$$

The result follows from the fact that $B_{p_{1}, q} B_{p^{\prime}, q}=1$ and $\left\|e_{q^{2}}\left(-t(\cdot)^{2}\right)\right\|_{p_{1}, q}=t^{-\frac{1}{2 p_{1}}} \alpha\left(p_{1}, q\right)$.

\section{Competing interests}

The authors declare that they have no competing interests.

\section{Authors' contributions}

All authors read and approved the final manuscript.

\section{Author details}

${ }^{1}$ College of Science and Human Studies at Hotat Sudair, Majmaah University, Riyadh, Saudi Arabia. ${ }^{2}$ Institut Préparatoire aux Études d'Ingénieur de El Manar, Tunis, Tunisia. ${ }^{3}$ Faculté des Sciences de Tunis, Université de Tunis El Manar, Tunis, 1060, Tunisia.

Received: 24 October 2012 Accepted: 20 May 2013 Published: 6 June 2013

\section{References}

1. Dhaouadi, L, El Kamel, J, Fitouhi, A: Positivity of the $q$-even translation and inequalities in q-Fourier analysis. Far East J. Math. Sci. 26, 137-154 (2007)

2. Bettaibi, N, Bouzeffour, F, Elmonser, HB, Binous, W: Elements of harmonic analysis related to the third basic zero order Bessel function. J. Math. Anal. Appl. 342, 1203-1219 (2008)

3. Gasper, G, Rahman, M: Basic Hypergeometric Series. Encyclopedia of Mathematics and its Application, vol. 35. Cambridge University Press, Cambridge (1990)

4. Jackson, FH: On a q-definite integrals. Q. J. Pure Appl. Math. 41, 193-203 (1910)

5. Fitouhi, A, Hamza, MM, Bouzeffour, F: The $q-J_{\alpha}$ Bessel function. J. Approx. Theory 115, 144-166 (2002) 
6. Koornwinder, TH, Swarttouw, RF: On q-analogues of the Fourier and Hankel transforms. Trans. Am. Math. Soc. 333 445-461 (1992)

7. Koelink, HT: The quantum group of plane motions and the Hahn-Exton q-Bessel functions. Duke Math. J. 76, 483-508 (1994)

8. Koelink, HT, Swarttouw, RF: A q-analogues of Graf's addition formula for the Hahn-Exton q-Bessel function. J. Approx. Theory 81(2), 260-273 (1995)

9. Abdi, WH: On certain q-difference equation and q-Laplace transform. R.S. Varma, F.N.I (1960)

10. Koornwinder, TH: Special functions and q-commuting variables. In: Ismail, MEH, Masson, DR, Rahman, M (eds.) Special Functions, $q$-Series and Related Topics. Fields Institute Communications vol. 14, pp. 131-166. Am. Math. Soc., Providence (1997). arXiv:q-alg/9608008

11. Daalhuis, ABO: Asymptotic expansions for q-Gamma, q-Exponential and q-Bessel Functions. J. Math. Anal. Appl. 186 896-913 (1994)

12. Fitouhi, A: Inégalité de Babenko et inégalité logarithmique de Sobolev pour l'opérateur de Bessel. C. R. Séances Acad. Sci., Sér. 1 Math. 305, 877-880 (1987)

doi:10.1186/1029-242X-2013-289

Cite this article as: Elmonser et al.: Inequalities related to the third Jackson $q$-Bessel function of order zero. Journal of Inequalities and Applications 2013 2013:289.

\section{Submit your manuscript to a SpringerOpen ${ }^{\ominus}$ journal and benefit from:}

- Convenient online submission

Rigorous peer review

- Immediate publication on acceptance

- Open access: articles freely available online

- High visibility within the field

- Retaining the copyright to your article 